\title{
ELEMENTS OF RINGS AND BANACH ALGEBRAS WITH RELATED SPECTRAL IDEMPOTENTS
}

\author{
N. CASTRO-GONZÁLEZ ${ }^{\unlhd}$ and J. Y. VÉLEZ-CERRADA
}

(Received 3 May 2004; revised 4 February 2005)

Communicated by J. Du

\begin{abstract}
Let $a^{\pi}$ denote the spectral idempotent of a generalized Drazin invertible element $a$ of a ring. We characterize elements $b$ such that $1-\left(b^{\pi}-a^{\pi}\right)^{2}$ is invertible. We also apply this result in rings with involution to obtain a characterization of the perturbation of EP elements. In Banach algebras we obtain a characterization in terms of matrix representations and derive error bounds for the perturbation of the Drazin inverse. This work extends recent results for matrices given by the same authors to the setting of rings and Banach algebras. Finally, we characterize generalized Drazin invertible operators $A, B \in \mathscr{B}(X)$ such that $\operatorname{pr}\left(B^{\pi}\right)=\operatorname{pr}\left(A^{\pi}+S\right)$, where pr is the natural homomorphism of $\mathscr{B}(X)$ onto the Calkin algebra and $S \in \mathscr{B}(X)$ is given.
\end{abstract}

2000 Mathematics subject classification: primary 16A32, 16A28, 15A09.

Keywords and phrases: Generalized Drazin inverse, spectral idempotent, EP elements, perturbation, Fredholm operators.

\section{Introduction}

The perturbations of the conventional and generalized Drazin inverse with equal eigenprojections at zero have been studied by Castro, Koliha and Wei in [4,5] for matrices and closed operators, respectively, by Rakočević [7] for elements in Banach algebras and by Koliha and Patricio [15] for elements of rings.

Recently, the authors [6] studied perturbations of the Drazin inverse of a square complex matrix $A$ for the case when the perturbed matrix $B$ has the eigenprojection at zero satisfying $B^{\pi}=A^{\pi}+S$, where $S$ is a given matrix. The results obtained therein can be applied to the case $S=0$ in order to obtain a characterization of matrices for which $B^{\pi}=A^{\pi}$ and, so, recover results of [4].

(C) 2006 Australian Mathematical Society $1446-7887 / 06 \$ A 2.00+0.00$ 
The main purpose of this paper is to investigate the perturbation of the generalized Drazin inverse for elements of rings and Banach algebras. In Section 3 we give a characterization of elements $a, b$ with spectral idempotents related by the condition that $1-\left(b^{\pi}-a^{\pi}\right)^{2}$ is invertible. In particular, if $b^{\pi}=a^{\pi}$ we recover [15, Theorem 6.1]. In Section 4 we apply this result in rings with involution to obtain a characterization of the perturbation of EP elements, that is, elements which have Drazin and MoorePenrose inverse and both coincide.

In several recent articles perturbations of the Drazin inverse were studied with a purpose to obtain explicit error bounds $[3,8,14,16-18]$. We work in Banach algebras in Section 5 and derive an upper bound of $\left\|b^{\mathrm{D}}-a^{\mathrm{D}}\right\| /\left\|a^{\mathrm{D}}\right\|$ in terms of $\left\|a^{\mathrm{D}}(b-a)\right\|$ and $\left\|b^{\pi}-a^{\pi}\right\|$. This estimation is sharper than the error bound given in [14, Theorem 2.1], and [3, Theorem 6.1] for matrices and closed operators, respectively.

Finally in Section 6, we give a characterization for bounded linear operators in Banach spaces, based on the main result of Section 3, of generalized Drazin invertible operators $A, B \in \mathscr{B}(X)$ such that their eigenprojections at zero satisfy $\operatorname{pr}\left(B^{\pi}\right)=$ $\operatorname{pr}\left(A^{\pi}+S\right)$, where $\mathrm{pr}$ is the natural homomorphism of $\mathscr{B}(X)$ onto the Calkin algebra. In particular, when $S=0$ we recover [7, Corollary 2.3].

\section{Preliminaries}

Let $\mathscr{R}$ be a ring with unit 1 . The commutant and the double commutant of an element $a \in \mathscr{R}$ are defined by

$$
\begin{aligned}
\operatorname{comm}(a) & =\{x \in \mathscr{R}: a x=x a\}, \\
\operatorname{comm}^{2}(a) & =\{x \in \mathscr{R}: x y=y x \text { for all } y \in \operatorname{comm}(a)\} .
\end{aligned}
$$

We denote by $\mathscr{R}^{-1}$ the group of invertible elements of $\mathscr{R}$. An element $a \in \mathscr{R}$ is quasinilpotent if $1+x a \in \mathscr{R}^{-1}$ for every $x \in \operatorname{comm}(a)$ [10]. The set of all quasinilpotent elements of $\mathscr{R}$ will be denoted by $\mathscr{R}^{\text {qnil }}$.

An element $a \in \mathscr{R}$ is said to have a generalized Drazin inverse if there exists $x \in \mathscr{R}$ such that

$$
x \in \operatorname{comm}^{2}(a), \quad x=a x^{2}, \quad a-a^{2} x \in \mathscr{R}^{\text {qnil }} .
$$

If $a$ has a generalized Drazin inverse, then the generalized Drazin inverse of $a$ is unique and is denoted by $a^{\mathrm{D}}$. If in the previous definition $a-a^{2} x$ is in fact nilpotent (which is equivalent to $a^{k+1} x=a^{k}$ where $k$ is the index of nilpotency), then $a^{\mathrm{D}}$ is the conventional Drazin inverse of $a$ and ind $(a)=k$ where ind $(a)$ is the Drazin index of $a$ [9]. Moreover, in this case the condition $x \in \operatorname{comm}^{2}(a)$ can be replaced with $x \in \operatorname{comm}(a)$. When ind $(a)=1$ the Drazin inverse of $a$ is called the group inverse, 
and is denoted by $a^{\mathrm{D}}=a^{\#}$. By $\mathscr{R}^{\mathrm{gD}}, \mathscr{R}^{\#}$ we denote the set of all generalized Drazin invertible and group invertible elements of $\mathscr{R}$, respectively.

The Drazin inverse in rings was originally defined by Drazin [9] for polar elements and generalized by Harte [10] to quasipolar elements. The generalized Drazin inverse was studied by Koliha [12] in Banach algebras and by Koliha and Patrício [15] in rings.

THEOREM 2.1. An element $a \in \mathscr{R}$ has a generalized Drazin inverse $a^{\mathrm{D}}$ if and only if there exists an idempotent $p \in \mathscr{R}$ such that

$$
p \in \operatorname{comm}^{2}(a), \quad a p \in \mathscr{R}^{\text {qnil }}, \quad a+p \in \mathscr{R}^{-1} .
$$

There is at most one idempotent $p$ such that conditions (2.2) hold. The unique idempotent $p$ is called a spectral idempotent of $a$ and it is denoted by $a^{\pi}$. It is known that

$$
a^{\mathrm{D}}=\left(a+a^{\pi}\right)^{-1}\left(1-a^{\pi}\right)=\left(1-a^{\pi}\right)\left(a+a^{\pi}\right)^{-1} \text { and } a^{\pi}=1-a a^{\mathrm{D}} .
$$

Now, we state two lemmas which will be needed in the next section. We recall that two idempotents elements $p_{1}$ and $p_{2}$ are called complementary provided that $p_{1}+p_{2}=1$.

LEMMA 2.2. Let $p_{1}, p_{2}$ be nonzero complementary idempotents in a ring $\mathscr{R}$. If $z \in \mathscr{R}$ commutes with $p_{2}$ and $1+p_{2} z \in \mathscr{R}^{-1}$, then

$$
z+p_{2} \in \mathscr{R}^{-1} \text { if and only if } p_{1} z+p_{2} \in \mathscr{R}^{-1} \text {. }
$$

PROOF. Since $z \in \mathscr{R}$ commutes with $p_{2}$, it follows that $\left(p_{1} z+p_{2}\right)\left(1+p_{2} z\right)=z+p_{2}$. Hence, the equivalence is verified because $1+p_{2} z \in \mathscr{R}^{-1}$.

Let $\mathscr{P}=\left(p_{1}, p_{2}\right)$ be a system of nonzero complementary idempotents elements in a ring $\mathscr{R}$. We define the set

$$
\mathscr{M}_{2}(\mathscr{R}, \mathscr{P})=\left\{\left(\begin{array}{ll}
x_{11} & x_{12} \\
x_{21} & x_{22}
\end{array}\right): x_{i j} \in p_{i} \mathscr{R} p_{j}, \text { for all } i, j \in\{1,2\}\right\}
$$

The set $\mathscr{M}_{2}(\mathscr{R}, \mathscr{P})$ is a ring with the usual matrix operations, with the unit $\left(\begin{array}{cc}p_{1} & 0 \\ 0 & p_{2}\end{array}\right)$ and the mapping $\phi: \mathscr{R} \rightarrow \mathscr{M}_{2}(\mathscr{R}, \mathscr{P})$ defined by

$$
\phi(a)=\left(\begin{array}{ll}
p_{1} a p_{1} & p_{1} a p_{2} \\
p_{2} a p_{1} & p_{2} a p_{2}
\end{array}\right)
$$

is a ring isomorphism. 
LEMMA 2.3. Let $p_{1}, p_{2}$ be nonzero complementary idempotents in a ring $\mathscr{R}$, and let $z \in \mathscr{R}$. If $p_{2} z p_{1}=0$ and $p_{i} z p_{i} \in\left(p_{i} \mathscr{R} p_{i}\right)^{-1}, i=1,2$, then $z \in \mathscr{R}^{-1}$.

PROOF. Since $p_{2} z p_{1}=0$, using the isomorphism $\phi: \mathscr{R} \rightarrow \mathscr{M}_{2}(\mathscr{R}, \mathscr{P})$ we obtain

$$
\phi(z)=\left(\begin{array}{cc}
p_{1} z p_{1} & p_{1} z p_{2} \\
0 & p_{2} z p_{2}
\end{array}\right)
$$

Since $p_{i} z p_{i} \in\left(p_{i} \mathscr{R} p_{i}\right)^{-1}, i=1,2$, it follows that the matrix $\phi(z)$ is invertible in $\mathscr{M}_{2}(\mathscr{R}, \mathscr{P})$ and, consequently, $z$ is invertible in $\mathscr{R}$.

The following sets will appear in the main theorem of the next section. If $a \in \mathscr{R}$, we define

$$
\begin{aligned}
a \mathscr{R} & =\{a x: x \in \mathscr{R}\}, & \mathscr{R} a & =\{x a: x \in \mathscr{R}\}, \\
a^{0} & =\{y \in \mathscr{R}: a y=0\}, & { }^{0} a & =\{y \in \mathscr{R}: y a=0\} .
\end{aligned}
$$

If $M \subset \mathscr{R}$, we define $M \mathscr{R}=\{m x: m \in M, x \in \mathscr{R}\}$ and $M^{0}=\{x \in \mathscr{R}: M x=\{0\}\}$. The sets $\mathscr{R} M$ and ${ }^{\circ} M$ can be defined similarly.

These sets have the following properties [11, Proposition 6]. We recall that an element $a \in \mathscr{R}$ is regular if there exists $x \in \mathscr{R}$ such that $a x a=a$, that is, if it has an inner inverse $x$.

PROPOSITION 2.4. Let $a, b \in \mathscr{R}$ be regular elements and $A, B \subset \mathscr{R}$. Then

(i) $a^{0}=(\mathscr{R} a)^{0}$.

(ii) $\left.\mathscr{R} a=9(\mathscr{R} a)^{0}\right)={ }^{0}\left(a^{0}\right)$.

(iii) $A \subset B$ implies ${ }^{0} A \supset{ }^{0} B$.

\section{Characterization of elements of rings with related spectral idempotents}

In this section we give a characterization of elements $a, b \in \mathscr{R}$ with spectral idempotents satisfying $b^{\pi}=a^{\pi}+s$, where $s$ is a given element of $\mathscr{R}$ such that $1-s^{2} \in \mathscr{R}^{-1}$. We remark that, for any $s \in \mathscr{R}^{\text {qnil }}$ we have $1-s^{2} \in \mathscr{R}^{-1}$. This research extends a recent work of the authors for matrices [6] to the setting of rings.

First we note the following properties involving the spectral idempotent $a^{\pi}$ and idempotent elements of the form $a^{\pi}+s$.

Proposition 3.1. Let $a \in \mathscr{R}^{\mathrm{D}}$ and $s \in \mathscr{R}$ such that $1-s^{2} \in \mathscr{R}^{-1}$. If $a^{\pi}+s$ is idempotent, then

(i) $a^{\pi}+s=(1-s)^{-1} a^{\pi}(1+s)=(1+s) a^{\pi}(1-s)^{-1}$; 
(ii) $1-a^{\pi}-s=(1-s)\left(1-a^{\pi}\right)(1+s)^{-1}=(1+s)^{-1}\left(1-a^{\pi}\right)(1-s)$;

(iii) if $r=(1+s) a^{\pi}+(1-s)\left(1-a^{\pi}\right)$, then $r \in \mathscr{R}^{-1}, r^{-1}=a^{\pi}(1-s)^{-1}$ $+\left(1-a^{\pi}\right)(1+s)^{-1}$ and $a^{\pi}+s=r a^{\pi} r^{-1}$.

Proof. Since $\left(a^{\pi}+s\right)^{2}=a^{\pi}+s$, we have $a^{\pi}+a^{\pi} s+s a^{\pi}+s^{2}=a^{\pi}+s$. Hence $a^{\pi}(1+s)=a^{\pi}+s-s a^{\pi}-s^{2}=(1-s)\left(a^{\pi}+s\right)$ and thus the first equality of (i) holds. The second equality of (i) and both equalities of (ii) can be deduced in a similar way.

Now, write $r=(1+s) a^{\pi}+(1-s)\left(1-a^{\pi}\right)$ and $t=a^{\pi}(1-s)^{-1}+\left(1-a^{\pi}\right)(1+s)^{-1}$. Applying properties (i) and (ii) we check that

$$
\begin{aligned}
r t & =(1+s) a^{\pi}(1-s)^{-1}+(1-s)\left(1-a^{\pi}\right)(1+s)^{-1} \\
& =\left(a^{\pi}+s\right)+\left(1-a^{\pi}-s\right)=1 .
\end{aligned}
$$

Analogously we can check that $t r=1$. Consequently, $t=r^{-1}$. On the other hand, $r a^{\pi} r^{-1}=(1+s) a^{\pi}(1-s)^{-1}=a^{\pi}+s$, where we apply property (i) in the last equality.

REMARK. Condition $\left(a^{\pi}+s\right)^{2}=a^{\pi}+s$ yields that $s^{2}\left(1-a^{\pi}\right)=\left(1-a^{\pi}\right) s^{2}=$ $\left(1-a^{\pi}\right) s\left(1-a^{\pi}\right)$ and $s^{2} a^{\pi}=a^{\pi} s^{2}=-a^{\pi} s a^{\pi}$.

We are now in a position to state the main result of this paper.

THEOREM 3.2. Let $a \in \mathscr{R}^{\mathrm{gD}}$ and let $s \in \mathscr{R}$ be such that $1-s^{2} \in \mathscr{R}^{-1}$. If $a^{\pi}+s$ is idempotent, then the following conditions on $b \in \mathscr{R}$ are equivalent:

(i) $b \in \mathscr{R}^{\mathrm{gD}}$, and $b^{\pi}=a^{\pi}+s$.

(ii) $a^{\pi}+s \in \operatorname{comm}^{2}(b),\left(a^{\pi}+s\right) b \in \mathscr{R}^{\text {qnil }}$, and $b+a^{\pi}+s \in \mathscr{R}^{-1}$.

(iii) $a^{\pi}+s \in \operatorname{comm}^{2}(b),\left(a^{\pi}+s\right) b \in \mathscr{R}^{\text {qnil }}$, and $1+s+a^{\mathrm{D}}(b-a) \in \mathscr{R}^{-1}$.

(iv) $b \in \mathscr{R}^{\mathrm{gD}}, 1+s+a^{\mathrm{D}}(b-a) \in \mathscr{R}^{-1}$, and $b^{\mathrm{D}}=\left(1+s+a^{\mathrm{D}}(b-a)\right)^{-1} a^{\mathrm{D}}(1-s)$.

(v) $b \in \mathscr{R}^{\mathrm{gD}}$, and $(1+s) b^{\mathrm{D}}-a^{\mathrm{D}}(1-s)=a^{\mathrm{D}}(a-b) b^{\mathrm{D}}$.

(vi) $b \in \mathscr{R}^{\mathrm{gD}}, a^{\pi}+s \in \operatorname{comm}(b)$, and $1-\left(b^{\pi}-a^{\pi}-s\right)^{2} \in \mathscr{R}^{-1}$.

(vii) $b^{\mathrm{D}}(1+s) \mathscr{R} \subset(1-s) a^{\mathrm{D}} \mathscr{R}$, and $\left(b^{\mathrm{D}}(1+s)\right)^{0} \subset\left((1-s) a^{\mathrm{D}}\right)^{0}$.

PROOF. (i) if and only if (ii): This equivalence follows by Theorem 2.1 .

(ii) if and only if (iii): Let $r=(1+s) a^{\pi}+(1-s)\left(1-a^{\pi}\right)$. By Proposition 3.1 (iii), we have that $r \in \mathscr{R}^{-1}$ and $a^{\pi}+s=r a^{\pi} r^{-1}$. Then the conditions (ii) and (iii) are equivalent to

(ii) $a^{\pi} \in \operatorname{comm}^{2}\left(r^{-1} b r\right), a^{\pi} r^{-1} b r \in \mathscr{R}^{\text {ynil }}$, and $r^{-1} b r+a^{\pi} \in \mathscr{R}^{-1}$.

(iii) $a^{\pi} \in \operatorname{comm}^{2}\left(r^{-1} b r\right), a^{\pi} r^{-1} b r \in \mathscr{R}^{\text {nil }}$, and $r a^{\pi}+a^{\mathrm{D}} b r \in \mathscr{R}^{-1}$. 
We will show that under the assumptions $a^{\pi} \in \operatorname{comm}^{2}\left(r^{-1} b r\right)$ and $a^{\pi} r^{-1} b r \in \mathscr{R}^{\text {qnil }}$,

$$
r^{-1} b r+a^{\pi} \in \mathscr{R}^{-1} \quad \text { if and only if } r a^{\pi}+a^{\mathrm{D}} b r \in \mathscr{R}^{-1} .
$$

Assume first that $r^{-1} b r+a^{\pi} \in \mathscr{R}^{-1}$. Let $z=r a^{\pi}+a^{\mathrm{D}} b r$. Then we can write

$$
z=a^{\pi} r a^{\pi}+a^{\mathrm{D}} r\left(r^{-1} b r+a^{\pi}\right)\left(1-a^{\pi}\right)+\left(1-a^{\pi}\right)\left(r+a^{\mathrm{D}} b r\right) a^{\pi} .
$$

We prove that $z$ is invertible. For this, first we observe that $p_{1}=1-a^{\pi}$ and $p_{2}=a^{\pi}$ are commuting complementary idempotents; we may assume that they are nonzero. Recall that $p_{1}, p_{2}$ commute with $a, r^{-1} b r$, and $1-s^{2}$. We can verify by a direct calculation that

$$
p_{2} z p_{1}=0, \quad p_{1} z p_{1}=p_{1}\left(a+p_{2}\right)^{-1} p_{1} r\left(r^{-1} b r+p_{2}\right) p_{1}, \quad \text { and } \quad p_{2} z p_{2}=p_{2} r p_{2} .
$$

In the ring $p_{1} \mathscr{R} p_{1}$ with unit $p_{1}$, we check that

$$
\left(p_{1}\left(a+p_{2}\right)^{-1} p_{1} r\left(r^{-1} b r+p_{2}\right) p_{1}\right)^{-1}=p_{1}\left(r^{-1} b r+p_{2}\right)^{-1}\left(1-s^{2}\right)^{-1}\left(a+p_{2}\right)^{-1} p_{1},
$$

and in the ring $p_{2} \mathscr{R} p_{2}$ with unit $p_{2}$, we check that $\left(p_{2} r p_{2}\right)^{-1}=p_{2}\left(1-s^{2}\right)^{-1} p_{2}$. According to Lemma 2.3, $z$ is invertible.

Conversely, assume that $r p_{2}+a^{\mathrm{D}} b r \in \mathscr{R}^{-1}$. First, we will prove that

$$
u=\left(a^{\mathrm{D}}+p_{2}\right)\left(1-s^{2}\right)\left(p_{1} r^{-1} b r+p_{2}\right)
$$

is invertible, which will imply that $p_{1} r^{-1} b r+p_{2} \in \mathscr{R}^{-1}$. Since $1+p_{2} r^{-1} b r \in \mathscr{R}^{-1}$ because $p_{2} r^{-1} b r \in \mathscr{R}^{\text {qnil }}$, Lemma 2.2 ensures that $r^{-1} b r+p_{2} \in \mathscr{R}^{-1}$.

We verify that $p_{2} u p_{1}=0$ and by a direct calculation, which takes into account the commutative relations mentioned above, we obtain that $p_{1} u p_{1}=p_{1}\left(r p_{2}+a^{\mathrm{D}} b r\right) p_{1}$ and $p_{2} u p_{2}=p_{2}\left(1-s^{2}\right) p_{2}$. Further,

$$
\begin{aligned}
\left(p_{1}\left(r p_{2}+a^{\mathrm{D}} b r\right) p_{1}\right)^{-1} & =p_{1}\left(r p_{2}+a^{\mathrm{D}} b r\right)^{-1} p_{1}, \\
\left(p_{2}\left(1-s^{2}\right) p_{2}\right)^{-1} & =p_{2}\left(1-s^{2}\right)^{-1} p_{2},
\end{aligned}
$$

in the rings $p_{1} \mathscr{R} p_{1}, p_{2} \mathscr{R} p_{2}$, respectively. By Lemma $2.3, u$ is invertible in $\mathscr{R}$, and consequently $r^{-1} b r+p_{2} \in \mathscr{R}^{-1}$.

(iii) implies (iv): Since condition (i) is true if and only if (ii) is true, and condition (ii) is true if and only if (iii) is true, we have $b^{\pi}=a^{\pi}+s$ and $1+s+a^{\mathrm{D}}(b-a) \in \mathscr{R}^{-1}$. By equation (2.3),

$$
\begin{aligned}
\left(1+s+a^{\mathrm{D}}(b-a)\right) b^{\mathrm{D}} & =\left(a^{\pi}+s+a^{\mathrm{D}} b\right)\left(1-\left(a^{\pi}+s\right)\right)\left(b+a^{\pi}+s\right)^{-1} \\
& =a^{\mathrm{D}} b\left(1-a^{\pi}-s\right)\left(b+a^{\pi}+s\right)^{-1} \\
& =a^{\mathrm{D}}(1-s) .
\end{aligned}
$$


Therefore (iv) holds.

(iv) implies (v): From $b^{\mathrm{D}}=\left(1+s+a^{\mathrm{D}}(b-a)\right)^{-1} a^{\mathrm{D}}(1-s)$ it follows that $(1+s) b^{\mathrm{D}}+a^{\mathrm{D}}(b-a) b^{\mathrm{D}}=a^{\mathrm{D}}(1-s)$ and thus (v) holds.

(v) implies (i): First multiply the equality given in (v) by $a^{\pi}$ from the left and then multiply the same equality by $b^{\pi}$ from the right to get

$$
a^{\pi}(1+s) b^{\mathrm{D}}=0 \quad \text { and } \quad a^{\mathrm{D}}(1-s) b^{\pi}=0 .
$$

Then we can write $a^{\pi}(1+s) b^{\mathrm{D}} b=a a^{\mathrm{D}}(1-s) b^{\pi}$. Hence,

$$
\begin{aligned}
a^{\pi}(1+s) & =\left(a^{\pi}(1+s)+\left(1-a^{\pi}\right)(1-s)\right) b^{\pi} \\
& =\left(a^{\pi}(1-s)^{-1}+\left(1-a^{\pi}\right)(1+s)^{-1}\right)\left(1-s^{2}\right) b^{\pi} .
\end{aligned}
$$

According to Proposition 3.1 (iii) and (i),

$$
\begin{aligned}
b^{\pi} & =\left(1-s^{2}\right)^{-1}\left((1+s) a^{\pi}+(1-s)\left(1-a^{\pi}\right)\right) a^{\pi}(1+s) \\
& =(1-s)^{-1} a^{\pi}(1+s)=a^{\pi}+s .
\end{aligned}
$$

(i) if and only if (vi): The right implication is obvious. We will check the left implication. From $1-\left(b^{\pi}-a^{\pi}-s\right)^{2} \in \mathscr{R}^{-1}$ it follows that $1-b^{\pi}+a^{\pi}+s \in \mathscr{R}^{-1}$ and $1+b^{\pi}-a^{\pi}-s \in \mathscr{R}^{-1}$. Since $a^{\pi}+s \in \operatorname{comm}(b)$ and $b b^{\pi}=b^{\pi} b$, we have $\left(a^{\pi}+s\right) b^{\pi}=b^{\pi}\left(a^{\pi}+s\right)$. Then $\left(1-a^{\pi}-s+b^{\pi}\right)\left(a^{\pi}+s\right)\left(1-b^{\pi}\right)=0$ and consequently $\left(a^{\pi}+s\right)\left(1-b^{\pi}\right)=0$. Hence, $a^{\pi}+s=\left(a^{\pi}+s\right) b^{\pi}$. On the other hand, we have $\left(1-b^{\pi}+a^{\pi}+s\right) b^{\pi}\left(1-a^{\pi}-s\right)=0$ and so $b^{\pi}\left(1-a^{\pi}-s\right)=0$. Therefore, $b^{\pi}=b^{\pi}\left(a^{\pi}+s\right)=a^{\pi}+s$.

(i) implies (vii): As $b^{\mathrm{D}} b=1-b^{\pi}=1-a^{\pi}-s$, we have $b b^{\mathrm{D}}=(1-s) a a^{\mathrm{D}}(1+s)^{-1}$ by Proposition 3.1 (ii). Then

$$
b^{\mathrm{D}}(1+s) \mathscr{R}=b b^{\mathrm{D}}(1+s) \mathscr{R}=(1-s) a a^{\mathrm{D}} \mathscr{R}=(1-s) a^{\mathrm{D}} \mathscr{R} .
$$

Similarly, $\mathscr{R} b^{\mathrm{D}}(1+s)=\mathscr{R}(1-s) a^{\mathrm{D}}$.

Since $b^{\mathrm{D}}(1+s)$ and $(1-s) a^{\mathrm{D}}$ are regular elements, by Proposition 2.4,

$$
\left(b^{\mathrm{D}}(1+s)\right)^{0}=\left(\mathscr{R} b^{\mathrm{D}}(1+s)\right)^{0}=\left(\mathscr{R}(1-s) a^{\mathrm{D}}\right)^{0}=\left((1-s) a^{\mathrm{D}}\right)^{0} .
$$

(vii) implies (i): As $\left(b^{\mathrm{D}}(1+s)\right)^{0} \subset\left((1-s) a^{\mathrm{D}}\right)^{0}$, by Proposition 2.4,

$$
\mathscr{R} b^{\mathrm{D}}(1+s)={ }^{0}\left(\left(b^{\mathrm{D}}(1+s)\right)^{0}\right) \supset^{0}\left(\left((1-s) a^{\mathrm{D}}\right)^{0}\right)=\mathscr{R}(1-s) a^{\mathrm{D}} .
$$

The above inclusion implies the consistency of the equation $x b b^{\mathrm{D}}(1+s)=(1-s) a^{\mathrm{D}} a$. Then $x b b^{\mathrm{D}}=(1-s) a^{\mathrm{D}} a(1+s)^{-1}=a a^{\mathrm{D}}-s$ by Proposition 3.1 (ii). This last equation is equivalent to $\left(a a^{\mathrm{D}}-s\right)\left(1-b b^{\mathrm{D}}\right)=0$. Thus

$$
a a^{\mathrm{D}}-s=\left(a a^{\mathrm{D}}-s\right) b b^{\mathrm{D}} \text {. }
$$


From $b^{\mathrm{D}}(1+s) \mathscr{R} \subset(1-s) a^{\mathrm{D}} \mathscr{R}$ the consistency of the equation

$$
b b^{\mathrm{D}}(1+s)=(1-s) a a^{\mathrm{D}} y
$$

follows, which in turn is equivalent to $\left(1-a a^{\mathrm{D}}\right)(1-s)^{-1} b b^{\mathrm{D}}=0$. Hence, by Proposition 3.1 (i), $(1+s)^{-1}\left(1-a a^{\mathrm{D}}-s\right) b^{\mathrm{D}} b=0$. Then, $b b^{\mathrm{D}}=\left(a a^{\mathrm{D}}-s\right) b^{\mathrm{D}} b$. Thus $b b^{\mathrm{D}}=a a^{\mathrm{D}}-s$ in view of (3.2), and (i) holds.

Specializing the equivalence of conditions (i)-(vi) to matrices we get the equivalence of conditions (a)-(b) and (d)-(f) given in [6, Theorem 2.3].

When we consider the case $s=0$ in the preceding theorem we get a characterization of elements of $\mathscr{R}$ with equal spectral idempotents. So, we recover [15, Theorem 6.1].

REMARK. If we do not assume condition $1-s^{2} \in \mathscr{R}^{-1}$, then we can find elements $a, b \in \mathscr{R}$ such that $b^{\pi}=a^{\pi}+s$, but $1+s+a^{\mathrm{D}}(b-a)$ is not an invertible element. We consider the real matrices $4 \times 4$,

$$
s=\left(\begin{array}{cccc}
-1 / 2 & 1 / 2 & 0 & 0 \\
1 / 2 & -1 / 2 & 0 & 0 \\
0 & 0 & 0 & 0 \\
0 & 0 & 0 & 0
\end{array}\right), \quad a=\left(\begin{array}{llll}
1 & 1 & 0 & 0 \\
1 & 1 & 0 & 0 \\
0 & 0 & 0 & 0 \\
0 & 0 & 0 & 0
\end{array}\right), \quad b=\left(\begin{array}{llll}
2 & 0 & 0 & 0 \\
0 & 2 & 0 & 0 \\
0 & 0 & 0 & 1 \\
0 & 0 & 0 & 0
\end{array}\right) .
$$

Then

$$
a^{\pi}=\left(\begin{array}{cccc}
1 / 2 & -1 / 2 & 0 & 0 \\
-1 / 2 & 1 / 2 & 0 & 0 \\
0 & 0 & 1 & 0 \\
0 & 0 & 0 & 1
\end{array}\right), \quad b^{\pi}=\left(\begin{array}{llll}
0 & 0 & 0 & 0 \\
0 & 0 & 0 & 0 \\
0 & 0 & 1 & 0 \\
0 & 0 & 0 & 1
\end{array}\right)
$$

and

$$
1+s+a^{\mathrm{D}}(b-a)=\left(\begin{array}{cccc}
1 / 2 & 1 / 2 & 0 & 0 \\
1 / 2 & 1 / 2 & 0 & 0 \\
0 & 0 & 1 & 0 \\
0 & 0 & 0 & 1
\end{array}\right)
$$

So, $b^{\pi}=a^{\pi}+s$ and $1+s+a^{\mathrm{D}}(b-a)$ is not invertible.

\section{Perturbation of EP elements in rings with involution}

Let $\mathscr{R}$ be a ring with involution. Recall that an element $x \in \mathscr{R}$ is the Moore-Penrose inverse of $a \in \mathscr{R}$ provided that

$$
x a x=x, \quad a x a=a, \quad(a x)^{*}=a x, \quad(x a)^{*}=x a .
$$


There is at most one $x$ such that conditions (4.1) hold. The unique $x$ is denoted by $a^{\dagger}$. The set of all Moore-Penrose invertible elements of $\mathscr{R}$ will be denoted by $\mathscr{R}^{\dagger}$.

An element $a$ is said to be $E P$ if $a \in \mathscr{R}^{\mathrm{gD}} \cap \mathscr{R}^{\dagger}$ and $a^{\mathrm{D}}=a^{\dagger}$. It is well-known that $a \in \mathscr{R}$ is EP if and only if $a a^{\dagger}=a^{\dagger} a$. Necessary and sufficient conditions for an element of a $\mathscr{C}^{*}$-algebras to commute with its Moore-Penrose inverse are given in [13].

In [15, Theorem 7.2 (i)-(ii)] it was established that $a \in \mathscr{R}$ is EP if and only if $a \in \mathscr{R}^{\#}$ and $a^{\pi}=\left(a^{\left.*^{\pi}\right)}\right.$. We will use this characterization and our main theorem to give equivalent conditions ensuring that if $a$ is EP then an element $b=a+e$ is again EP with spectral idempotent $b^{\pi}=a^{\pi}+s$ where $s$ is given.

THEOREM 4.1. Let $\mathscr{R}$ be a ring with involution and let $s \in \mathscr{R}$ be such that $1-s^{2} \in \mathscr{R}^{-1}$. If $a$ is $E P$ and $a^{\pi}+s$ is idempotent, then the following conditions on $b=a+e \in \mathscr{R}$ are equivalent:

(i) $b$ is EP and $b^{\pi}=a^{\pi}+s$.

(ii) $s^{*}=s, e\left(a^{\#} a-s\right)-a s=e=\left(a^{\#} a-s\right) e-s a$, and $1+s+a+e-a^{\#} a \in \mathscr{R}^{-1}$.

(iii) $s^{*}=s, e\left(a^{\#} a-s\right)-a s=e=\left(a^{\#} a-s\right) e-s a$, and $1+s+a^{\#} e \in \mathscr{R}^{-1}$.

(iv) $b \in \mathscr{R}^{{ }^{\mathrm{D}}} \cap \mathscr{R}^{\dagger}$, and $b^{\dagger}=b^{\mathrm{D}}=\left(1+s+a^{\dagger} e\right)^{-1} a^{\dagger}(1-s)$.

(v) $b \in \mathscr{R}^{\mathrm{gD}} \cap \mathscr{R}^{\dagger}, b^{\dagger}=b^{\mathrm{D}}$, and $(1+s) b^{\dagger}-a^{\dagger}(1-s)=-a^{\dagger} e b^{\dagger}$.

PROOF. Since $a$ is EP, we have $a \in \mathscr{R}^{\#}$ and $\left(a^{*}\right)^{\pi}=\left(a^{\pi}\right)^{*}=a^{\pi}$. Now, if $b^{\pi}=a^{\pi}+s$ then $\left(b^{*}\right)^{\pi}=\left(b^{\pi}\right)^{*}=\left(a^{\pi}\right)^{*}+s^{*}=a^{\pi}+s^{*}$. Hence, condition (i) is equivalent to

(i) $\quad b \in \mathscr{R}^{\#}, s$ is selfadjoint, and $b^{\pi}=a^{\pi}+s$.

Using $a^{\pi}=1-a a^{\#}$ and $a a^{\pi}=0$, conditions (ii) and (iii) can be written in the following equivalent form

(ii) $s^{*}=s,(a+e)\left(a^{\pi}+s\right)=0=\left(a^{\pi}+s\right)(a+e)$, and $a^{\pi}+s+a+e \in \mathscr{R}^{-1}$. (iii) $^{\prime} s^{*}=s,(a+e)\left(a^{\pi}+s\right)=0=\left(a^{\pi}+s\right)(a+e)$, and $1+s+a^{\#} e \in \mathscr{R}^{-1}$.

Applying Theorem 3.2 we conclude the equivalence of conditions (i)-(v) of the theorem.

Given $s \in \mathscr{R}$, we obtain the following characterization of elements $a \in \mathscr{R}$ such that the spectral idempotents of $a$ and $a^{*}$ satisfy $\left(a^{*}\right)^{\pi}=a^{\pi}+s$.

THEOREM 4.2. Let $\mathscr{R}$ be a ring with involution and let $s \in \mathscr{R}$ be such that $1-s^{2} \in \mathscr{R}^{-1}$. Then the following conditions on $a \in \mathscr{R}$ are equivalent:

(i) $a^{*} \in \mathscr{R}^{\#}$, and $\left(a^{*}\right)^{\pi}=a^{\pi}+s$.

(ii) $a^{*} \in \mathscr{R}^{\mathrm{gD}},\left(a^{*}\right)^{*} a^{*}=a a^{\mathrm{D}}-s$, and $(1+s) a^{*}=a^{\mathrm{D}} a a^{*}$.

(iii) $a^{*}\left(a^{\mathrm{D}} a-s\right)=a^{*}=\left(a^{\mathrm{D}} a-s\right) a^{*}, 1+s+a^{*}-a^{\mathrm{D}} a \in \mathscr{R}^{-1}$, and $s^{2}+s=$ $s a a^{\mathrm{D}}+a a^{\mathrm{D}} s$. 
(iv) $s^{2}+s=s a a^{\mathrm{D}}+a a^{\mathrm{D}} s, 1+s+a^{\mathrm{D}}\left(a^{*}-a\right) \in \mathscr{R}^{-1}$, and $a^{*}\left(a^{\mathrm{D}} a-s\right)=a^{*}=$ $\left(a^{\mathrm{D}} a-s\right) a^{*}$.

Proof. From $a^{\pi}=1-a^{\mathrm{D}} a$ we easily see that the conditions (ii)-(iv) can be formulated in the following equivalent form

(ii) $\quad\left(a^{*}\right)^{\pi}=a^{\pi}+s$, and $a^{*}\left(a^{\pi}+s\right)=0$.

(iii) $\left(a^{\pi}+s\right)^{2}=a^{\pi}+s, a^{*}\left(a^{\pi}+s\right)=\left(a^{\pi}+s\right) a^{*}=0$, and $a^{*}+a^{\pi}+s \in \mathscr{R}^{-1}$.

(iv) $\left(a^{\pi}+s\right)^{2}=a^{\pi}+s, a^{*}\left(a^{\pi}+s\right)=\left(a^{\pi}+s\right) a^{*}=0$, and $1+s+a^{\mathrm{D}}\left(a^{*}-a\right) \in \mathscr{R}^{-1}$.

Now, we apply Theorem 3.2 (i)-(iii) to $a^{*}$ in place of $b$.

If we choose $s=0$ in the preceding theorem, then condition (i) is equivalent to the fact that $a$ is EP. So, in this case, we obtain a characterization of EP elements in rings with involution. In particular, for matrices we recover [4, Theorem 5.2].

\section{Perturbation of Drazin invertible elements in Banach algebras}

Let $\mathscr{B}$ be a complex Banach algebra with unit 1 . The generalized Drazin inverse for elements of Banach algebras is defined as in (2.1) where the condition of double commutativity $x \in \operatorname{comm}^{2}(a)$ can be replaced by $x \in \operatorname{comm}(a)$. Let $\mathscr{B}^{g D}$ denote the set of all elements in $\mathscr{B}$ which have a generalized Drazin inverse.

The equivalence of conditions (i)-(vii) in Theorem 3.2 is also valid in Banach algebras. Moreover, in (ii) and (iii), the condition $a^{\pi}+s \in \operatorname{comm}^{2}(b)$ can be relaxed to $a^{\pi}+s \in \operatorname{comm}(b)$.

In this section, first we will give a new characterization of elements $a, b \in \mathscr{B}^{\mathrm{gD}}$ such that $1-\left(b^{\pi}-a^{\pi}\right)^{2}$ is invertible in terms of its matrix representation. Second, we will apply Theorem 3.2 to get a norm estimation of the perturbation of the Drazin inverse.

Let $a \in \mathscr{B}^{\mathrm{gD}}$. We consider a system of complementary idempotents $\mathscr{P}=\left(p_{1}, p_{2}\right)$, where $p_{1}=1-a^{\pi}$ and $p_{2}=a^{\pi}$. The set of matrices $\mathscr{M}_{2}(\mathscr{B}, \mathscr{P})$, defined as in Section 2, is a Banach algebra with the usual matrix operations, with the unit $\left(\begin{array}{cc}p_{1} & 0 \\ 0 & p_{2}\end{array}\right)$. The mapping $\phi: \mathscr{B} \rightarrow \mathscr{M}_{2}(\mathscr{B}, \mathscr{P})$ defined by

$$
\phi(a)=\left(\begin{array}{ll}
p_{1} a p_{1} & p_{1} a p_{2} \\
p_{2} a p_{1} & p_{2} a p_{2}
\end{array}\right)
$$

is an isometric Banach algebra isomorphism [2]. This fact will allow us to work with matrix representations of elements in $\mathscr{B}$ by identifying $a \in \mathscr{B}$ with its image $\phi(a) \in \mathscr{M}_{2}(\mathscr{B}, \mathscr{P})$. For brevity, we denote $\mathscr{B}_{i}=p_{i} \mathscr{B} p_{i}$ which is an algebra with unit $p_{i}, i=1,2$. 
If $a \in \mathscr{B}^{\mathrm{gD}}$, then it has a matrix representation

$$
a=\left(\begin{array}{cc}
a_{1} & 0 \\
0 & a_{2}
\end{array}\right), \quad a_{1} \in \mathscr{B}_{1}^{-1} \quad \text { and } \quad a_{2} \in \mathscr{B}_{2}^{\text {qnil }},
$$

and the generalized Drazin inverse of $a$ is given by

$$
a^{\mathrm{D}}=\left(\begin{array}{cc}
a_{1}^{-1} & 0 \\
0 & 0
\end{array}\right)
$$

where the inverse $a_{1}^{-1}$ is taken in $\mathscr{B}_{1}$.

It is straightforward to prove the following characterization which involves matrix representation of elements with equal spectral idempotent.

LEMmA 5.1. Let $a \in \mathscr{B}^{\mathrm{D} D}$. Then the following conditions on $b \in \mathscr{B}$ are equivalent:

(i) $b \in \mathscr{B}^{\mathrm{gD}}$ and $b^{\pi}=a^{\pi}$.

(ii) $b=\left(\begin{array}{cc}b_{1} & 0 \\ 0 & b_{2}\end{array}\right), b_{1} \in \mathscr{B}_{1}^{-1}$ and $b_{2} \in \mathscr{B}_{2}^{\text {qnil }}$, where $\mathscr{B}_{i}=p_{i} \mathscr{B} p_{i}$ for $i=1,2$.

THEOREM 5.2. Let $a \in \mathscr{B}^{\mathrm{gD}}$ and $s \in \mathscr{B}$ such that $1-s^{2} \in \mathscr{B}^{-1}$. If $a^{\pi}+s$ is idempotent, then the following conditions on $b \in \mathscr{B}$ are equivalent:

(i) $b \in \mathscr{B}^{\mathrm{gD}}$ and $b^{\pi}=a^{\pi}+s$.

(ii) The matrix representation for $b$ is

$b=\left(\begin{array}{cc}p_{1}(1-s) p_{1} & p_{1} s p_{2} \\ -p_{2} s p_{1} & p_{2}(1+s) p_{2}\end{array}\right)\left(\begin{array}{cc}b_{1} & 0 \\ 0 & b_{2}\end{array}\right)\left(\begin{array}{cc}p_{1} & -p_{1} s(1+s)^{-1} p_{2} \\ p_{2} s(1-s)^{-1} p_{1} & p_{2}\end{array}\right)$,

$b_{1} \in \mathscr{B}_{1}^{-1}$ and $b_{2} \in \mathscr{B}_{2}^{\text {qnil }}$, where $\mathscr{B}_{i}=p_{i} \mathscr{B} p_{i}$ for $i=1,2$.

ProOF. Consider $r=(1+s) a^{\pi}+(1-s)\left(1-a^{\pi}\right)=1-s+2 s a^{\pi}$. Under the hypothesis of this theorem we apply Proposition 3.1 (iii) to conclude that $r$ is nonsingular, $r^{-1}=a^{\pi}(1-s)^{-1}+\left(1-a^{\pi}\right)(1+s)^{-1}=\left(1-s+2 a^{\pi} s\right)\left(1-s^{2}\right)^{-1}$, and $r\left(a^{\pi}+s\right) r^{-1}=a^{\pi}$. Moreover, $r$ and $r^{-1}$ have the following matrix representations:

$$
\begin{aligned}
r & =\left(\begin{array}{cc}
p_{1}(1-s) p_{1} & p_{1} s p_{2} \\
-p_{2} s p_{1} & p_{2}(1+s) p_{2}
\end{array}\right) \text { and } \\
r^{-1} & =\left(\begin{array}{cc}
p_{1} & -p_{1} s(1+s)^{-1} p_{2} \\
p_{2} s(1-s)^{-1} p_{1} & p_{2}
\end{array}\right) .
\end{aligned}
$$

Now, define $\tilde{b}=r^{-1} b r$. Then $b^{\pi}=a^{\pi}+s$ if and only if $\tilde{b}^{\pi}=r^{-1}\left(a^{\pi}+s\right) r=a^{\pi}$. Applying Lemma 5.1, we get that $b^{\pi}=a^{\pi}+s$ is equivalent to having for $\tilde{b}$ the matrix representation $\tilde{b}=\left(\begin{array}{cc}b_{1} & 0 \\ 0 & b_{2}\end{array}\right)$, where $b_{1} \in \mathscr{B}_{1}^{-1}$ and $b_{2} \in \mathscr{B}_{2}^{\text {qnil }}$. Consequently, the equivalence between (i) and (ii) holds because $b=r \tilde{b} r^{-1}$. 
Theorem 3.2, when it is considered in the setting of Banach algebras, enables us to obtain the following upper bound of $\left\|b^{\mathrm{D}}-a^{\mathrm{D}}\right\| /\left\|a^{\mathrm{D}}\right\|$.

THEOREM 5.3. Let $a, b \in \mathscr{B}^{\mathrm{gD}}$. If $\left\|b^{\pi}-a^{\pi}\right\|+\left\|a^{\mathrm{D}}(b-a)\right\|<1$, then

$$
\frac{\left\|b^{\mathrm{D}}-a^{\mathrm{D}}\right\|}{\left\|a^{\mathrm{D}}\right\|} \leq \frac{\left\|a^{\mathrm{D}}(b-a)\right\|+2\left\|b^{\pi}-a^{\pi}\right\|}{1-\left\|b^{\pi}-a^{\pi}\right\|-\left\|a^{\mathrm{D}}(b-a)\right\|} .
$$

Proof. Write $s=b^{\pi}-a^{\pi}$. Since $\|s\|<1$, we have $1-s, 1+s \in \mathscr{B}^{-1}$. Now, by Theorem $3.2(\mathrm{v}),(1+s) b^{\mathrm{D}}-a^{\mathrm{D}}(1-s)=a^{\mathrm{D}}(a-b) b^{\mathrm{D}}$. Applying the norm to $b^{\mathrm{D}}-a^{\mathrm{D}}=-\left(s+a^{\mathrm{D}}(b-a)\right)\left(a^{\mathrm{D}}+\left(b^{\mathrm{D}}-a^{\mathrm{D}}\right)\right)-a^{\mathrm{D}} s$ we get

$$
\left\|b^{\mathrm{D}}-a^{\mathrm{D}}\right\| \leq\left(\|s\|+\left\|a^{\mathrm{D}}(b-a)\right\|\right)\left(\left\|a^{\mathrm{D}}\right\|+\left\|b^{\mathrm{D}}-a^{\mathrm{D}}\right\|\right)+\left\|a^{\mathrm{D}}\right\|\|s\|
$$

and from here the result follows.

We can combine this estimation with an upper bound of $\left\|b^{\pi}-a^{\pi}\right\|$ in order to get explicit error bounds of the perturbation of the Drazin inverse. An estimation of $\left\|b^{\pi}-a^{\pi}\right\|$ in terms of the gap between subspaces has been obtained in [14, Section 4] for matrices and in [3, Section 6] for bounded operators.

\section{Operators with eigenprojections essentially differing in a given term}

This section is motivated by recent results of Rakočević [7] which characterize the perturbation of the generalized Drazin invertible operators with essentially equal eigenprojections at zero.

We denote by $\mathscr{B}(X)$ and $\mathscr{K}(X)$ the set of all bounded and compact linear operators on an infinite dimensional complex Banach space $X$, respectively. The Calkin algebra over $X$ is defined as the quotient algebra $\mathscr{C}(X)=\mathscr{B}(X) / \mathscr{K}(X)$, with the norm

$$
\|T+\mathscr{K}(X)\|=\inf _{K \in \mathscr{K}(X)}\|T+K\| .
$$

We will use pr to denote the natural homomorphism of $\mathscr{B}(X)$ onto $\mathscr{C}(X)$. Then, if $T \in \mathscr{B}(X)$ we have $\operatorname{pr}(T)=T+\mathscr{K}(X)$. Let $r_{e}(T)$ denote the essential spectral radius of $T$, that is, $r_{e}(T)=\lim \left\|\operatorname{pr}\left(T^{n}\right)\right\|^{1 / n}$. An operator $T \in \mathscr{B}(X)$ is a Riesz operator if and only if $r_{e}(T)=0$, which is equivalent to $\operatorname{pr}(T)$ being quasinilpotent in $\mathscr{C}(X)$. By $\mathscr{R}(X)$ we denote the set of all Riesz operator in $\mathscr{B}(X)$.

Recall that an operator $T \in \mathscr{B}(X)$ is Fredholm if the range space of $T, \mathrm{R}(T)$, is closed and the kernel of $T$ and the quotient $X / \mathrm{R}(T)$ are both finite dimensional. The collection of Fredholm operators on $X$ is denoted by $\Phi(X)$.

By the Atkinson theorem [1, Theorem 3.2.8], $T$ is Fredholm if and only if $\operatorname{pr}(T)$ is an invertible element of $\mathscr{C}(X)$. 
Based on Theorem 3.2, we characterize the perturbation of the generalized Drazin invertible operators with eigenprojections essentially differing in a given bounded operator.

Corollary 6.1. Let $A \in \mathscr{B}(X)^{\mathrm{gD}}$ and $S \in \mathscr{B}(X)$ such that $I-S^{2}$ is invertible. If $A^{\pi}+S$ is idempotent, then the following conditions on $B \in \mathscr{B}(X)$ are equivalent:

(i) $B \in \mathscr{B}(X)^{\mathrm{gD}}$, and $\operatorname{pr}\left(B^{\pi}\right)=\operatorname{pr}\left(A^{\pi}+S\right)$.

(ii) $\operatorname{pr}\left(\left(A^{\pi}+S\right) B\right)=\operatorname{pr}\left(B\left(A^{\pi}+S\right)\right),\left(A^{\pi}+S\right) B \in \mathscr{R}(X)$, and $B+A^{\pi}+S \in \Phi(X)$.

(iii) $I+S+A^{\mathrm{D}}(B-A) \in \Phi(X), \operatorname{pr}\left(\left(A^{\pi}+S\right) B\right)=\operatorname{pr}\left(B\left(A^{\pi}+S\right)\right)$, and $\left(A^{\pi}+S\right) B \in$ $\mathscr{R}(X)$.

(iv) $B \in \mathscr{B}(X)^{\mathrm{gD}}, I+S+A^{\mathrm{D}}(B-A) \in \Phi(X)$, and $B^{\mathrm{D}}=C A^{\mathrm{D}}(I-S)+K$, where $C \in \mathscr{B}(X)$ is a Fredholm inverse of $I+S+A^{\mathrm{D}}(B-A)$, and $K \in \mathscr{K}(X)$.

(v) $B \in \mathscr{B}(X)^{\mathrm{g} D}$, and $(I+S) B^{\mathrm{D}}-A^{\mathrm{D}}(I-S)=A^{\mathrm{D}}(A-B) B^{\mathrm{D}}+L, L \in \mathscr{K}(X)$.

If $S=0$ in the above corollary, then we get [7, Corollary 2.3].

\section{Acknowledgement}

The authors are grateful to the referee, whose careful reading of the paper and valuable suggestions improved the presentation.

\section{References}

[1] S. R. Caradus, W. E. Pfaffenberger and B. Yood, Calkin algebras and algebras of operators on Banach spaces, Lecture Notes in Pure and Applied Mathematics 9 (Marcel Dekker, New York, 1974).

[2] N. Castro-González and J. J. Koliha, 'New additive results for the $g$-Drazin inverse', Proc. Roy. Soc. Edinburgh Sect. A 134 (2004), 1085-1097.

[3] N. Castro-González, J. J. Koliha and V. Rakočević, 'Continuity and general perturbation of the Drazin inverse for closed operators', Abstr. Appl. Anal. 6 (2002), 335-347.

[4] N. Castro-González, J. J. Koliha and Y. Wei, 'Perturbation of the Drazin inverse for matrices with equal eigenprojection at zero', Linear Algebra Appl. 312 (2000), 181-189.

[5] — - Error bounds for perturbation of the Drazin inverse of closed operators with equal spectral projections', Appl. Anal. 81 (2002), 915-928.

[6] N. Castro-González and J. Y. Vélez-Cerrada, 'Characterizations of matrices which eigenprojections at zero are equal to a fixed perturbation', Appl. Math. Comput. 159 (2004), 613-623.

[7] V. Rakočević, 'Koliha-Drazin invertible operators and commuting Riesz perturbations', Acta Sci. Math. (Szeged) 68 (2002), 291-301.

[8] V. Rakočević and Y. Wei, 'The perturbation theory for the drazin inverse and its aplications II', $J$. Aust. Math. Soc. 69 (2000), 1-9. 
[9] M. P. Drazin, 'Pseudo-inverses in associate rings and semigroups', Amer. Math. Monthly 65 (1958), 506-514.

[10] R. E. Harte, 'On quasinilpotents in rings', Panamer. Math. J. 1 (1991), 10-16.

[11] R. E. Hartwig, 'Block generalized inverses', Arch. Ration. Mech. Anal. 61 (1976), 197-251.

[12] J. J. Koliha, 'A generalized Drazin inverse', Glasgow Math. J. 38 (1996), 367-381.

[13] _ 'Elements of $C^{*}$-algebras commuting with their Moore-Penrose inverse', Studia Math. 139 (2000), 81-90.

[14] - 'Error bound for a general perturbation of the Drazin inverse', Appl. Math. Comput. 126 (2002), 181-185.

[15] J. J. Koliha and P. Patricio, 'Elements of rings with equal spectral idempotents', J. Aust. Math. Soc. 72 (2002), 137-152.

[16] X. Li and Y. Wei, 'An improvement on the perturbation of the group inverse and oblique projection', Linear Algebra Appl. 338 (2001), 53-66.

[17] Y. Wei and X. Li, 'An improvement on the perturbation bounds for the Drazin inverse', Numer. Linear Algebra Appl. 10 (2003), 563-575.

[18] Y. Wei and G. Wang, 'The perturbation theory for the Drazin inverse and its aplications', Linear Algebra Appl. 258 (1997), 179-186.

\section{Facultad de Informática}

Universidad Politécnica de Madrid

28660 Boadilla del Monte

Madrid

Spain

e-mail: nieves@fi.upm.es, jyvelezc@hotmail.com 